\title{
A new compact Design of a three-dimensional Ionization Profile Monitor (IPM)
}

\author{
Heiko Breede*, Hans-Jürgen Grabosch, Martin Sachwitz, \\ Deutsches Elektronen Synchrotron, Platanenallee 6, 15738 Zeuthen, Germany
}

\begin{abstract}
The Free Electron Laser FLASH at the German Electron Synchrotron (DESY) in Hamburg is a linear accelerator, which uses superconducting technology to produce soft x-ray laser light ranging from 4,1 to $45 \mathrm{~nm}$. To ensure the operation stability of FLASH, monitoring of the beam is mandatory. Among various detectors located at the beam pipe, two Ionization Profile Monitors (IPM) detect the lateral $\mathrm{x}$ and y position changes. The functional principle of the IPM is based on the detection of electrons, generated by interaction of the photon beam with the residual gas in the beam line. The newly designed IPM enables the combined determination of the FEL's horizontal and vertical position as well as the beam's profile. This is made possible by a compact monitor, consisting of a cage in a vacuum chamber, two microchannel plates (MCP) and two structural repeller plates with toggled electric fields at the opposite sides of the MCPs. The electrons created by the FEL beam, drift in a homogenous electrical field towards the respective micro-channel plate, which produces an image of the beam profile on an attached phosphor screen. A CCD camera for each MCP in combination with a computer is used for the evaluation. This indirect detection scheme operates over a wide dynamic range and allows the detection of the center of gravity and the shape of the photon beam without affecting the FEL beam. Exact knowledge of the path taken by the electrons permits a recursive determination of the beam position. Within a beam variance of less than $10 \mathrm{~mm}$, an accuracy better than $\pm 8 \mathrm{um}$ seems to be possible.
\end{abstract}

Keywords: Ionization Profile Monitor, laser monitor, soft x-ray laser light detector, laser detector, Residual gas detector, detector, FEM 


\section{INTRODUCTION}

To ensure a smooth operation of the free electron laser FLASH at DESY Hamburg, numerous detectors for the precise measurement of the electron and laser beam are necessary. The great advantage of the here described Ionization Profile Monitor (IPM) is an undisturbed determination of the position and intensity distribution of the laser beam.

\section{Measuring principle of an Ionizing Projection Monitor (IPM)}

The FLASH laser beam with a variable wavelength from 4.1 to $45 \mathrm{~nm}$ is located in an Ultra High Vacuum (UHV) beam pipe. Despite the vacuum a certain amount of residual gases still exist. If the laser beam hits a residual gas atom, it becomes ionized and charged electrons and ions are created. By means of a homogeneous electric field, these electrons and ions can be deflected in a rectilinear way towards the micro-channel plate (MCP). Here, the impacting particles create an avalanche of secondary electrons in the micro tubes of the MCP and are being visualized on the phosphorscreen. These results in an image of the intensity-dependent laser beam profile (see Figure 1).

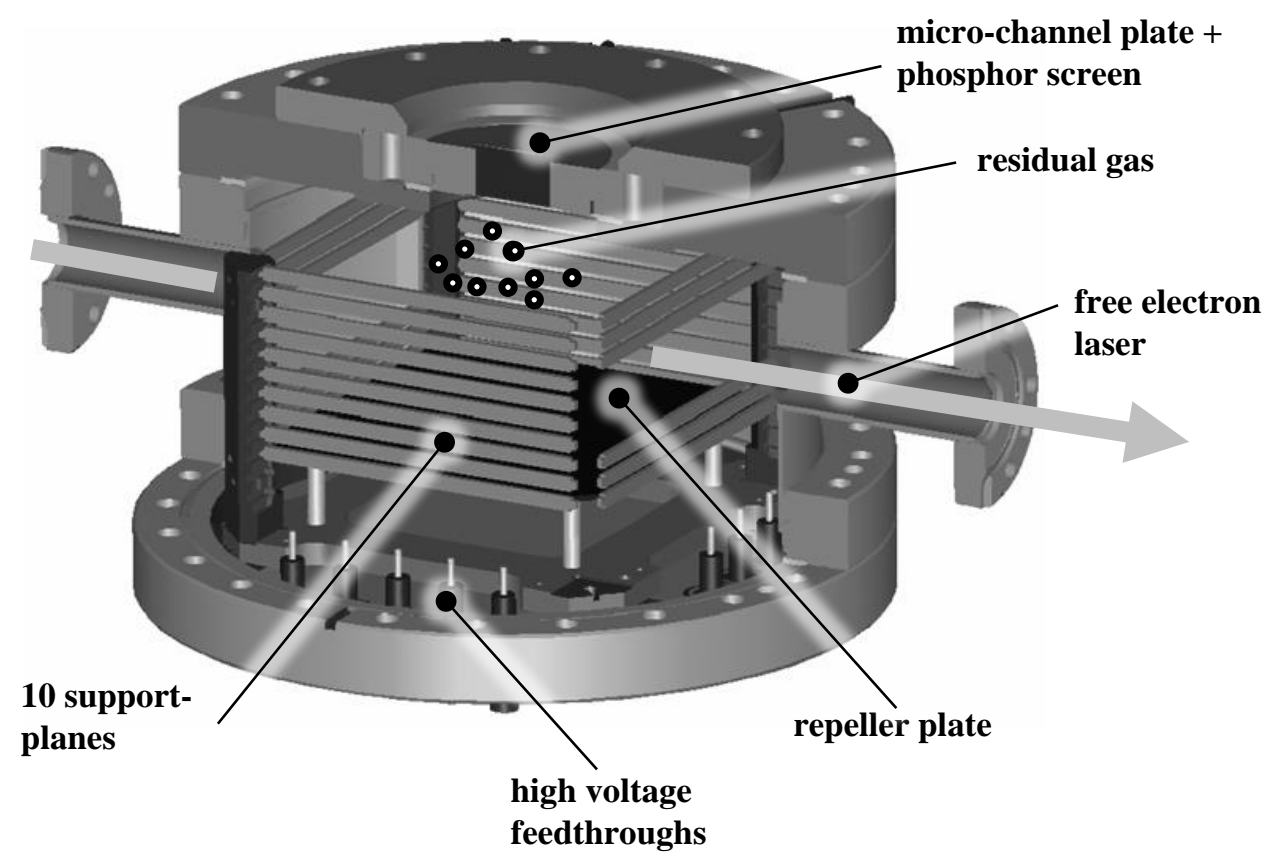

Figure 1. An Ionisation Profil Monitors as implemented in FLASH [1] [5].

\section{Conventional set up}

Figure 2 shows an IPM module for the laser beam position measurement as implemented in FLASH [1] [5]. Problems and disadvantages of this design are the following:

- The large size of the monitor (approx. $400 \mathrm{~mm}$ x $300 \mathrm{~mm}$ x $200 \mathrm{~mm}$ ) results in an insufficient homogeneity of the electrical field applied. Therefore the exact path of the electrons or ions from the origin of creation to the $\mathrm{MCP}$ is unknown and the spatial resolution is in the order of about \pm 50 microns.

- The size makes the IPM harder to manufacture and more expensive due to the high number of high voltage feedthroughs.

- To detect the horizontal and vertical parameters of the laser beam (3D) two consecutive detectors have to be implemented with perpendicular orientation to each other demanding large space. 
- To take a look at the single bunches rather than just examining the whole train, the IPM needs a time resolution of at least $100 \mathrm{~ns}$, the conventional set is not capable of.

\section{A NEW DESIGN OF THE IONIZATION PROFILE MONITOR}

In order to tackle the challenges described above the following design is proposed:

- Unification of the separate horizontal and vertical monitors with an alternating homogeneous electric field.

- A special cage protects the area of interest from electrical stray fields to ensure an optimal homogenous electric field.

- Decreasing the size of the device to $100 \mathrm{~mm}$ x $100 \mathrm{~mm}$ x $100 \mathrm{~mm}$ (or less) while at the same time reducing the applied electrical voltage with the appropriate low cost feedthroughs.

- With the Finite Element Method (FEM) a comparison of different residual gas particles is performed concerning their trajectories in the electric field.

- This procedure offers an optimization of the design by simulating the trajectory of the particles in the electrical field with the deflection caused by the inhomogeneity of the field. Varying the CAD monitor model helps finding out the best possible determination of the laser beam position.

Following the principles described above in figure 3 the resulting new design of a 3D detector is presented. The inner cage with a measure of $100 \mathrm{~mm} \times 100 \mathrm{~mm}$ x $100 \mathrm{~mm}$ consists of equal sized pads and two $28 \mathrm{~mm}$ diameter holes for the passage of the beam. Figure 3 shows 30 different electrical potentials are needed for the homogeneous field. 
micro-

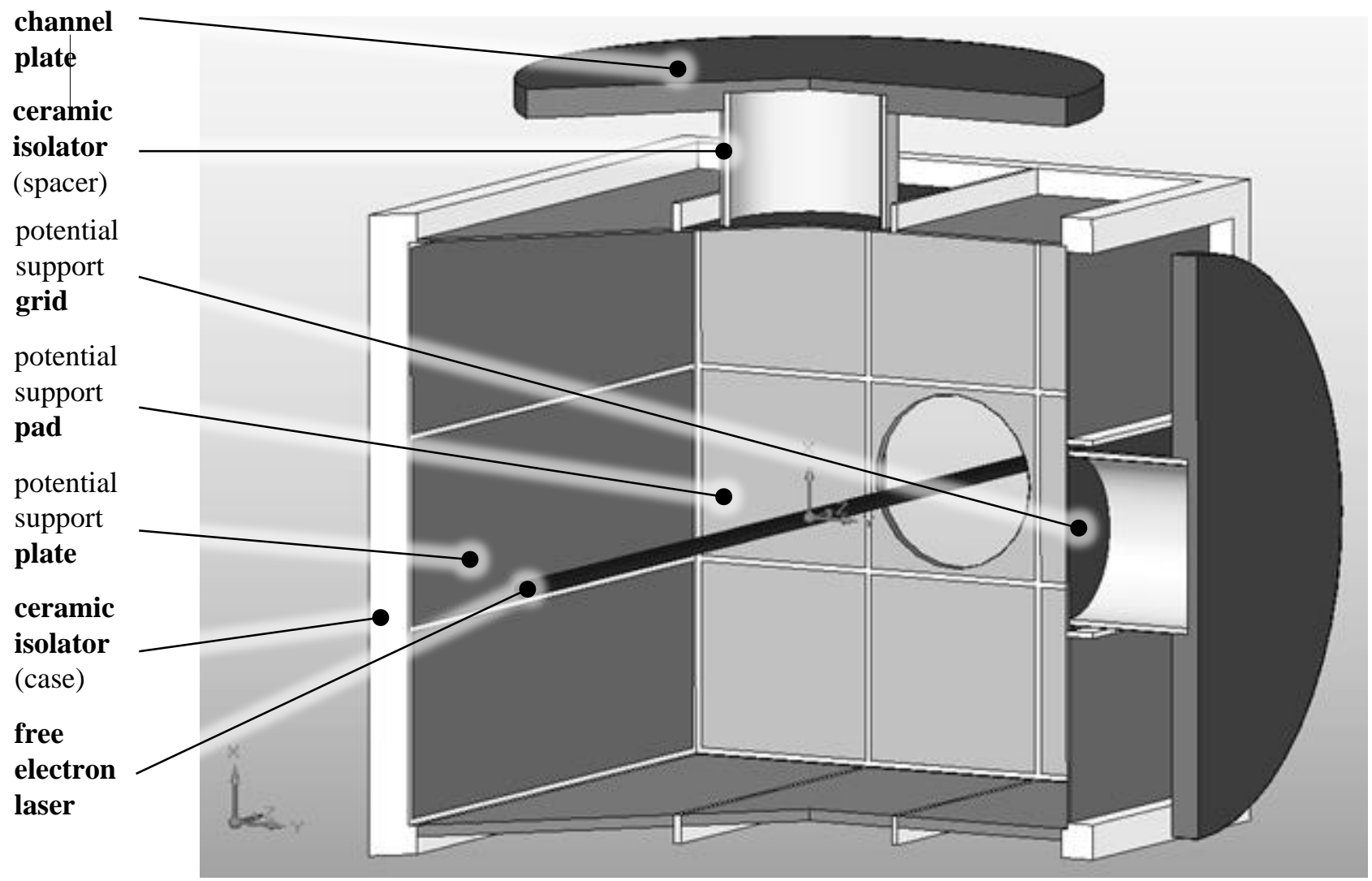

Figure 2. Schematically design of the 3D Ionization Profile Monitor.

\section{FEM ANALYSIS}

To analyze the homogeneity of the electric field and to determine the trajectory of different particles obtained with the design described above, a FEM analysis was carried out using ANSYS 14 modules workbench [2] and classic [2]. In the simulations different variations and possible future developments of the design were included directly from the CADmodel.

\section{Potential ratios}

Simulation studies performed with the workbench module package proved the potential ratios, as can be seen in figure 4 , to be optimal for a homogeneous electric field and hence for a straight flight of particles. To avoid inhomogeneity of the electric field no potential difference is applied between the MCP and the end of the cage. Due to the sufficient high speed of the particles at the end of the cage inertia leads the particles through the last part of their way. Since the MCP has a diameter of merely $28 \mathrm{~mm}$, only in the marked "area of interest" the electric field must be homogeneous. Also, the expected beam variation in $\mathrm{X}$ or $\mathrm{Y}$ is below $\pm 5 \mathrm{~mm}$. Homogeneity in a larger space does not result in a higher spatial resolution.

The electrically conductive potential supporting points are assumed as being an ideal conductor with equal potential at any point. The permittivity of the ceramic was assigned to $\varepsilon=6$ and of the vacuum to $\varepsilon=1$. 


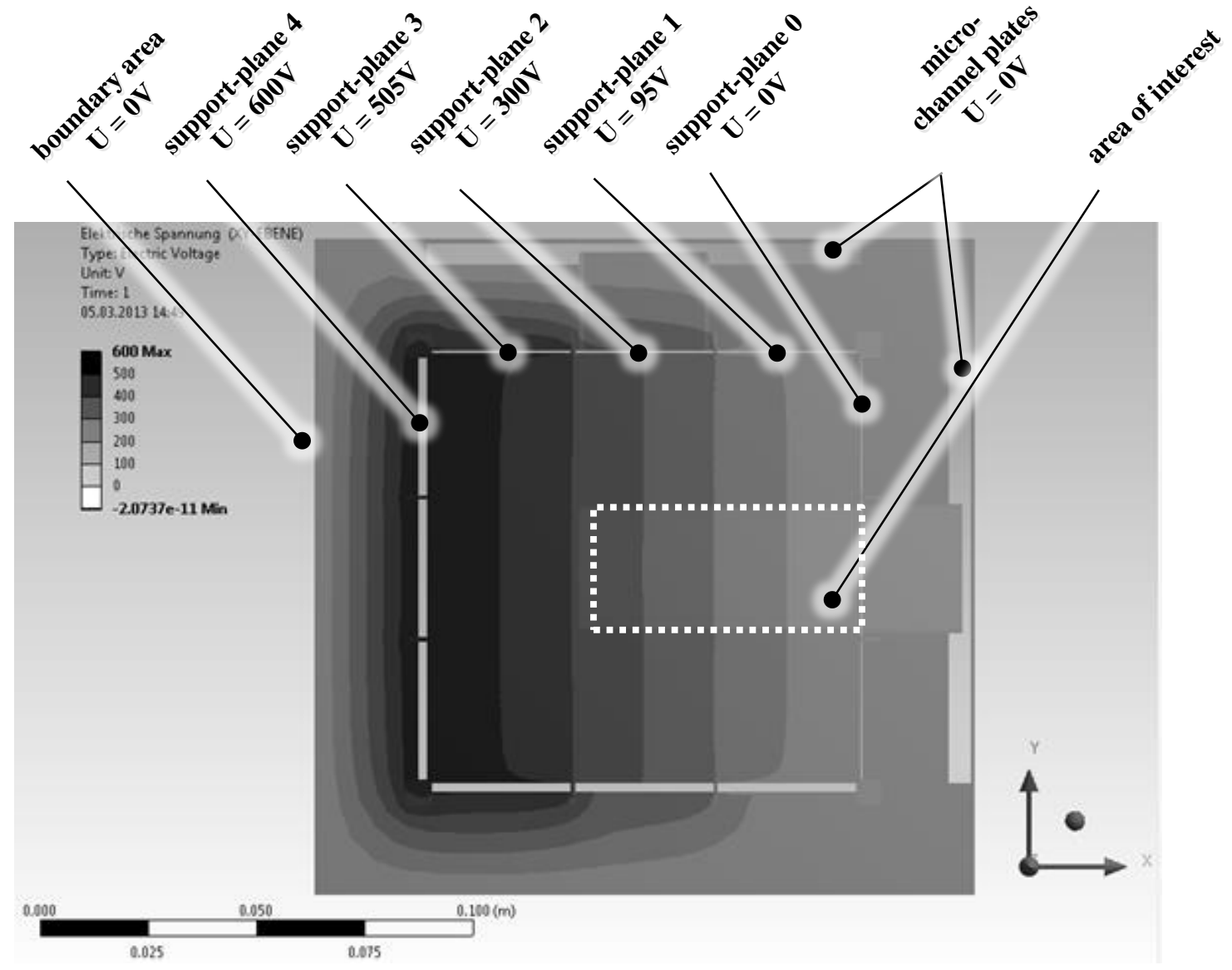

Figure 3. Equipotential lines of the electrical field.

\section{Animation of toggling the electric field}

The following Video shows an animation of toggling the electric field. It shows that the field is homogen in two directions in the area of interest.

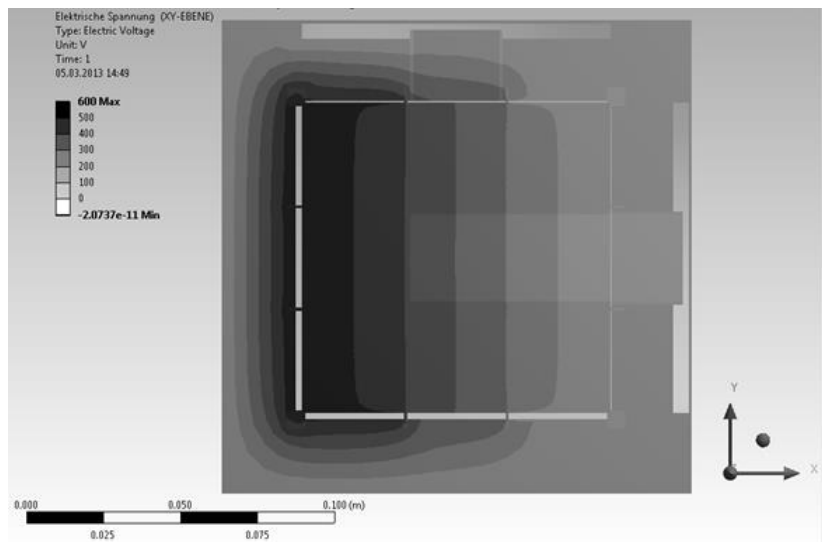

Video 1. Animation of toggling the electric field: http://dx.doi.org/doi.number.goes.here 


\section{Electrical field strength}

The equipotential lines correspond directly to the electrical field lines, as can be seen on figure 5. Inside the cage, in the area of interest, the vectors of the electric field lines are absolutely straight. Outside of the cage the nonlinear lines are too weak to influence the trajectory at all.

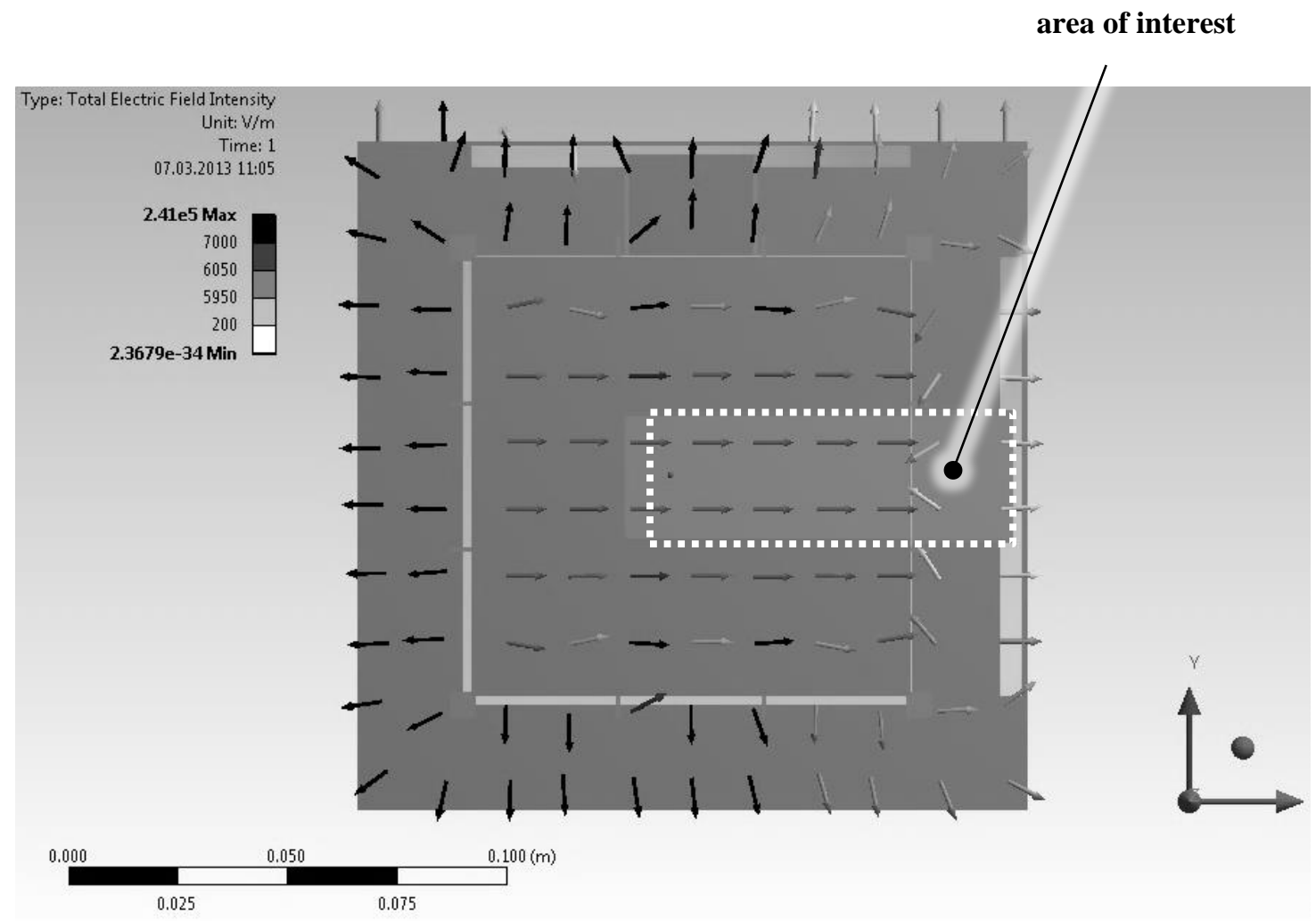

\section{Trajectories of electrons and ions}

Figure 4. Intensity distribution of the electric field.

To compare whether electrons or ions are better suited for the beam position detection, one has to compare trajectory and speed from a meaningful chosen point of creation. The boundary conditions of the trajectory determination are strongly idealized. A change of the field due to the charged electrons and ions and particle collisions are considered as negligible. This approximation is reasonable as the beam is located in the Ultra High Vacuum (UHV) beam pipe. Relevant influences are not expected. Terrestrial gravitation is taken into account with a starting speed of $0.987 \mathrm{~m} / \mathrm{s}$ towards the negative Y-axis. The starting points, as well as all determined trajectories are shown in Figure 6. 


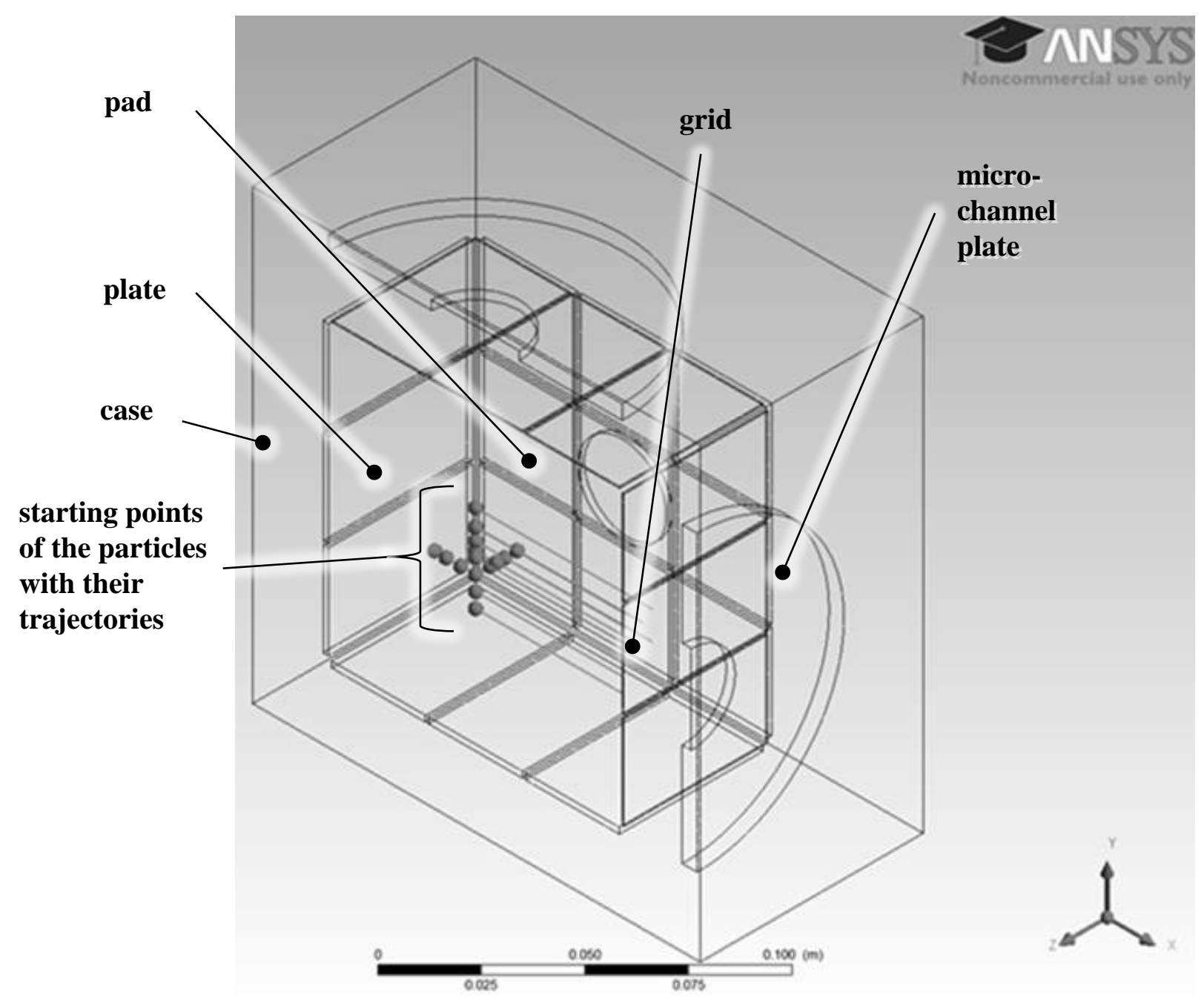

Figure 5. Starting points of the particles with their trajectories. 
In order to study the different behavior of the electrons and ions the coordinate $(x=-7.07 \mathrm{~mm} ; \mathrm{y}=7.07 \mathrm{~mm} ; \mathrm{z}=-7.07$ $\mathrm{mm}$ ) was chosen $\left[7.07 \mathrm{~mm}=\frac{10 \mathrm{~mm}}{\sqrt{2}}\right]$. The following particles have been compared with each other:

Table 1. Electron and ion types.

\begin{tabular}{l|r|r} 
Name & mass [kg] & charge [pC] \\
\hline $\mathrm{e}$ & $9.11 \mathrm{E}-31$ & $1.60 \mathrm{E}-07$ \\
$\mathrm{~N}^{2+}$ & $2.33 \mathrm{E}-26$ & $3.20 \mathrm{E}-07$ \\
$\mathrm{Ra}^{+}$ & $3.69 \mathrm{E}-25$ & $1.60 \mathrm{E}-07$
\end{tabular}

\section{Animations of the electron trajectories}

The following Video 2 shows the trajectory of electrons at the two switching states. The red and orange marked line represents the beam which ionizes the residual atoms. Beam-variation of $15 \mathrm{~mm}$ means, that the coordinates of the beam are $\mathrm{x}=10.61 \mathrm{~mm}$ and $\mathrm{y}=10.61 \mathrm{~mm}$ along to the $\mathrm{Z}$-axis $\left(10.61 \mathrm{~mm}=\frac{15 \mathrm{~mm}}{\sqrt{2}}\right)$. The electrons are represented as balls.

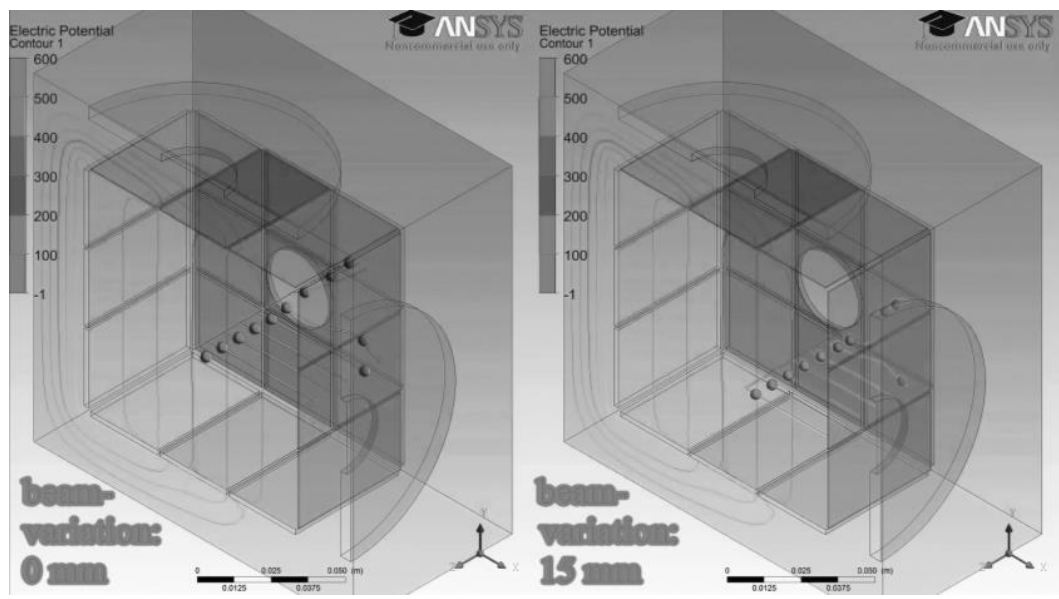

Video 2. Animation of the electrons trajectory 3D: http://dx.doi.org/doi.number.goes.here

Video 3 describes the deviation of the electrons caused by the inhomogeneous electric field. In this video electrons are represented as fishes for a better illustration of their trajectory

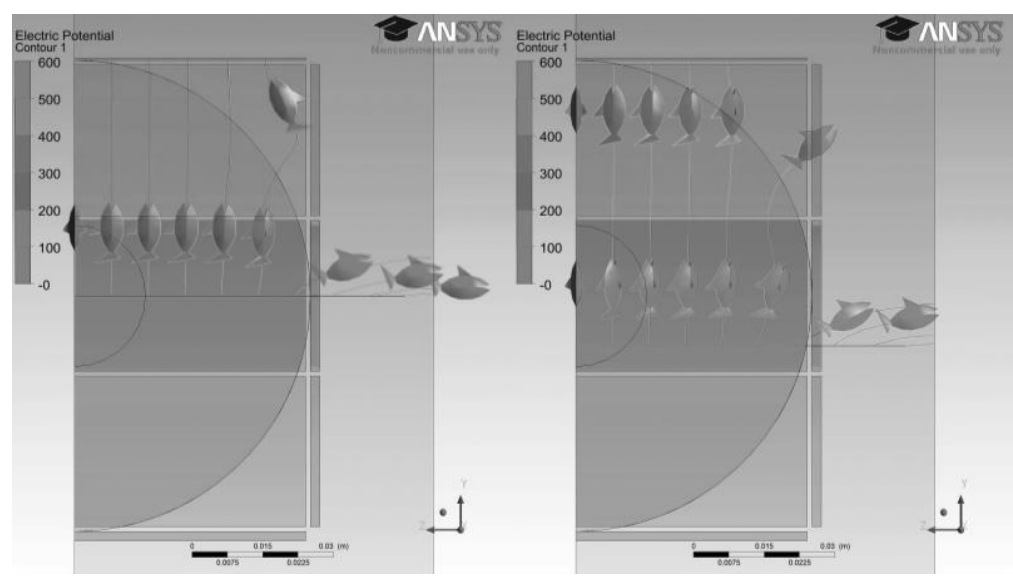

Video 3. Animation of the electrons trajectory 2D: http://dx.doi.org/doi.number.goes.here 


\section{Analysis of trajectories}

The trajectory of the particles is primarily relevant in Y direction. Any displacements concerning the Z-axis can be considered less important as long as they are sufficiently small. As can be seen in figure 7, the trajectory of the electrons and ions is compared with the simple addition of the vectors of the electrical field strength (streamlines). Inertia has been included in the equation. Figure 7 shows the resulting Y-displacement of the electrons and ions from the starting point until the end of the cage as a result of the inhomogeneity of the field. The final displacement is specified.

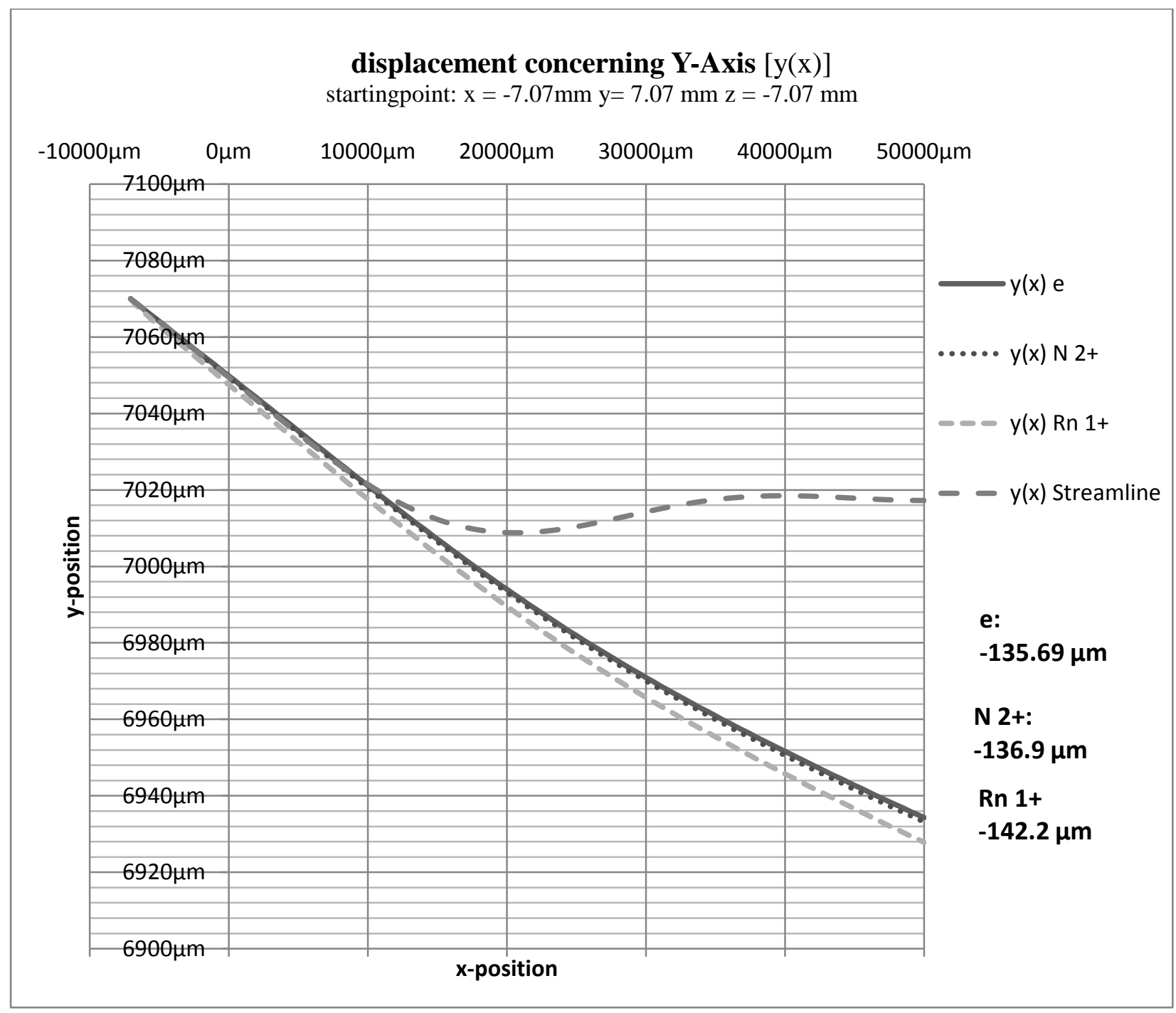

Figure 6. Position displacement in Y direction of electron and ions starting from the same origin.

The differences in the displacements are caused primarily by different speed and accelerations. A high acceleration in Xdirection suppresses the influence of the starting speed of the electrons and ions. Due to inertia the different lines show, once a direction has been chosen it is maintained. As can be seen from the streamline, the inhomogeneity of the field is strongest in the middle of the assembly module. Furthermore the speed variation in figure 8 shows the course of the speed of the electrons and ions. 


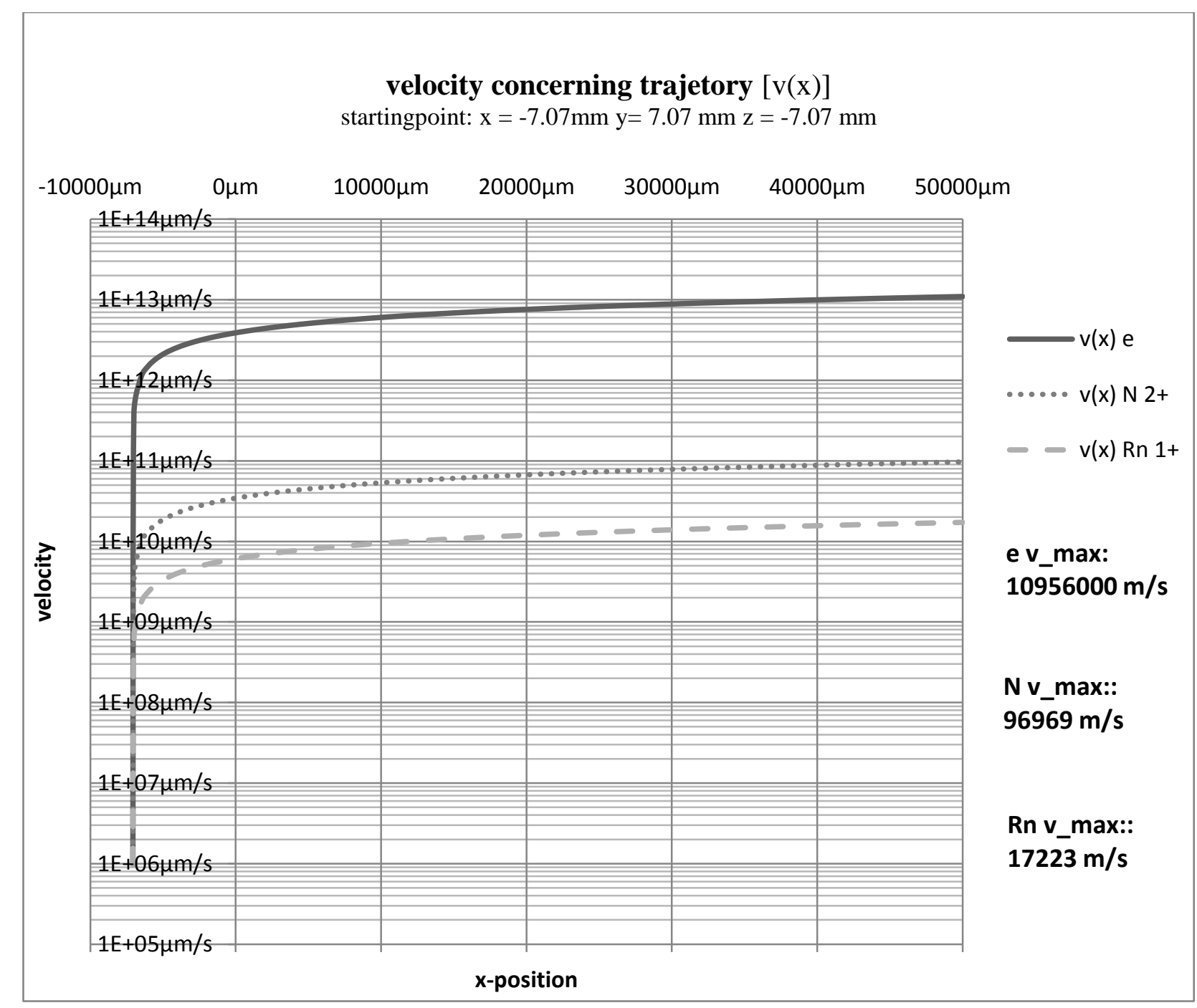

Figure 7. Velocity of electron and ions along their trajectory.

The high velocity of the electrons in particular makes it possible to use devices with the highest recording frequency. In the simulation merely 10.4 ns elapse between the start of the electron and the passing of the wall of the assembly module through the grid. They reach a maximum speed of about 11 million m/s. It takes the nitrogen ions 1'170 ns for the same route, which is 100 times slower. Thus every $10 \mathrm{~ns}$ a change of the beam can be detected. If the electrons are used, the electric field can be toggled quickly. This makes it possible to detect an image of the laser beam in a very short period of time, created by the fast moving electrons.

Taking this into account, for future IPM developments, one should always use electrons for beam detection. Only the electrons attain a very high time resolution of the bunches. In this case, a phosphor-screen with a much lower decay time than the normally used (about $1 \mathrm{~ms}$ ) is mandatory. A combination of a fast phosphor-screen [4] with a high speed camera can deliver a time resolution in the order of $100 \mathrm{~ns}$. 


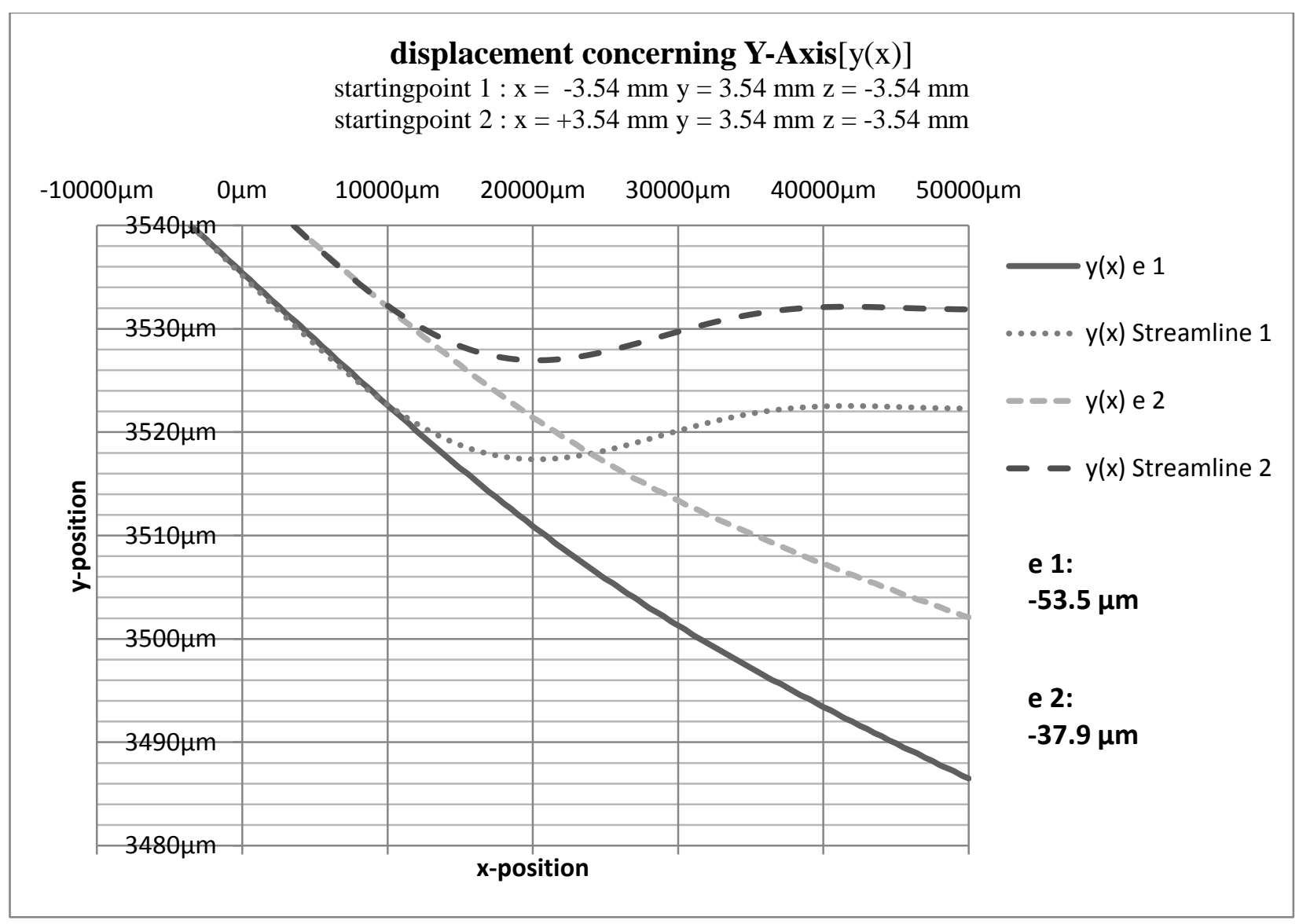

Figure 8: Displacement in Y direction of an electron starting from $3.54 \mathrm{~mm}$ behind and in front of the middle point.

In figure 9 the first electron trajectory starting behind the middle point of the detector from $\mathrm{x}=-3.54 \mathrm{~mm}$ is indicated with the number 1 and the second electron trajectory with the origin in front of the middle point $\mathrm{x}=3.54 \mathrm{~mm}(3.54 \mathrm{~mm}$ $=\frac{5 \mathrm{~mm}}{\sqrt{2}}$ ) with the number 2 . Trajectories with starting points $3.54 \mathrm{~mm}$ apart from the middle point of the detector are extreme but realistic assumptions for a routine operation of the IPM. The electron starting at $\mathrm{x}=3.54 \mathrm{~mm}$ shows a stronger curvature than the electron started at $\mathrm{x}=-3.54$, which can be explained by a shorter path length in the inhomogeneity. Of course, the displacements are lower if the distance to the end of the cage becomes shorter. From figure 9 one can see the shifts in the trajectory are within a manageable level. In average we have a displacement of -45.7 $\mu \mathrm{m}$ with a maximal error of $7.8 \mu \mathrm{m}$

$$
\frac{|-53.5 \mu \mathrm{m}|-|37.9 \mu \mathrm{m}|}{2}=7.8 \mu \mathrm{m}
$$

Since the path of the electron is precisely known a software-based interpolation of the image with the true value of the beam seems to be possible. 
The following table shows the displacements of the electrons concerning the $\mathrm{Y}$ - and Z-axis from the respective starting point until passing the grid. The final velocity is shown as well.

Table 2. Relation of the displacement of electrons.

\begin{tabular}{|c|c|c|c|c|c|c|c|}
\hline $\begin{array}{l}\text { start point } \\
\text { offset } \\
\text { concerning }\end{array}$ & $\begin{array}{c}\text { start point } \\
\text { nr. }\end{array}$ & $\begin{array}{c}\text { X- } \\
\text { coordinate } \\
{[\mathrm{mm}]}\end{array}$ & $\begin{array}{c}\mathrm{Y}- \\
\text { coordinate } \\
{[\mathrm{mm}]}\end{array}$ & $\begin{array}{c}\mathrm{Z}- \\
\text { coordinate } \\
{[\mathrm{mm}]}\end{array}$ & $\begin{array}{c}\text { displacement } \\
\text { concerning } \\
\text { Y-Axis } \\
{[\mu \mathrm{m}]}\end{array}$ & $\begin{array}{c}\text { displacement } \\
\text { concerning } \\
\text { Z-Axis } \\
{[\mu \mathrm{m}]}\end{array}$ & $\begin{array}{c}\text { final } \\
\text { velocity } \\
\text { [million } \\
\mathrm{m} / \mathrm{s} \text { ] }\end{array}$ \\
\hline \multirow{4}{*}{$\mathrm{X}$-Axis } & 1 & 0 & 0 & 0 & 0.54 & -10.81 & 10.245 \\
\hline & 2 & -5 & 0 & 0 & 0.53 & -11.08 & 10.753 \\
\hline & 3 & -10 & 0 & 0 & 0.49 & -10.49 & 11.238 \\
\hline & 4 & -14 & 0 & 0 & 0.43 & -9.30 & 11.612 \\
\hline \multirow{5}{*}{ Y-Axis } & 5 & 0 & -5 & 0 & 64.60 & -10.12 & 10.246 \\
\hline & 6 & 0 & 5 & 0 & -62.40 & -10.41 & 10.246 \\
\hline & 7 & 0 & -10 & 0 & 124.50 & -8.72 & 10.247 \\
\hline & 8 & 0 & 10 & 0 & -122.30 & -9.33 & 10.247 \\
\hline & 9 & 0 & 14 & 0 & -167.00 & -7.37 & 10.249 \\
\hline \multirow{2}{*}{ Z-Axis } & 10 & 0 & 0 & -5 & 2.90 & -211.20 & 10.244 \\
\hline & 11 & 0 & 0 & -10 & 2.69 & -429.00 & 10.241 \\
\hline \multirow{5}{*}{$\mathrm{X}, \mathrm{Y} \& \mathrm{Z}$-Axis } & 12 & -3.54 & 3.54 & -3.54 & -53.50 & -139.10 & 10.607 \\
\hline & 13 & 3.54 & 3.54 & -3.54 & -37.90 & -128.60 & 9.870 \\
\hline & 14 & -7.07 & 7.07 & -7.07 & -135.69 & -269.40 & 10.956 \\
\hline & 15 & 7.07 & 7.07 & -7.07 & -68.70 & -231.70 & 9.481 \\
\hline & 16 & -9.89 & 9.89 & -9.89 & -219.30 & -360.00 & 11.133 \\
\hline
\end{tabular}

As can be derived from figure 9 and table 2 the deviation of the electron path from the electrical field line is not symmetrically with respect to the middle axis of the detector. This can be explained with the unsymmetrical design.

Certainly the micro-channel plate located at the upper part of the assembly module has an influence on the electrical field lines and therefore on the path of the electrons. Compared with the opposite outer case the MCP is closer located to the cage and as consequence electrons with a starting point $\mathrm{Y}<0$ are stronger deflected. Highest accuracy of the device is obtained when the beam is in the middle of the device. Here is the lowest displacement of the electrons.

The defined startup speed, simulating the influence of the gravity, has no noticeable impact. 


\section{CONCLUSION}

A unification of two IPMs into one compact module has the advantage of a reduced assembly size combined with an increased accuracy of the measured beam parameters. A special designed cage shields the area of interest from unwanted influence of stray fields. The voltage applied for the different grids and potential supporting pads is relatively low. Nevertheless the small diameter of the cage of $100 \mathrm{~mm} \times 100 \mathrm{~mm} \times 100 \mathrm{~mm}$ allows a strong electrical field leading to very high acceleration and speed of the electrons created. Due to the much higher speed of the electrons compared to the ions, time resolution of about $100 \mathrm{~ns}$ can be achieved. The simulations have shown a spatial resolution in the order of \pm $45 \mu \mathrm{m}$ can be obtained with a laser beam fluctuation of $\pm 5 \mathrm{~mm}$.

Beam fluctuation bigger than the diameter of the area of interest deteriorates the result. Since highest accuracy is obtained in the middle of the device an axis modular motion control system should be taken into consideration.

The application of the finite-elements-method poses the possibility to increase precision of future IPMs enormously. In a realistic way the results of the simulations describe the trajectories of different electrons and ions following the electrical field. The displacement of the path of the electrons and ions from the electrical field lines can be precisely predicted. As shown in this paper, applying this procedure the spatial resolution can be improved by a factor of five.

\section{REFERENCES}

[1] M. Sachwitz, A. Hofmann, S. Pauliuk, K. Tiedtke, H. Wabnitz, "Proc. of EPAC08”, 1266-1268 (2008).

[2] ANSYS 14 Workbench und Classic, www.ansys.de.

[3] Solid Edge ST3, http://www.plm.automation.siemens.com/de_de/products/velocity/solidedge.

[4] Phosphor-screen P47, Hamamatsu Photonics Company, www.hamamatsu.com.

[5] Miesner, J., Sachwitz, M., Markert, M., Sternberger, R., Tiedtke, K., Hofmann, A., „An Ionization Profile Monitor for the determination of the FLASH photon beam parameter", Nucl. Instr. and Meth. A 635, S104S107 (2011). 